\title{
Erratum: A model for holographic QCD in the Veneziano limit at finite temperature and density
}

\author{
T. Alho, ${ }^{a, b}$ M. Järvinen, ${ }^{c}$ K. Kajantie, ${ }^{b}$ E. Kiritsis,,${ }^{c, d, e, 1}$ C. Rosen ${ }^{c}$ and K. Tuominen ${ }^{f, b}$ \\ ${ }^{a}$ Department of Physics, University of Jyväskylä, \\ P.O. Box 35, FI-40014 Jyväskylä, Finland \\ ${ }^{b}$ Helsinki Institute of Physics, University of Helsinki, \\ P.O. Box 64, FI-00014 Helsinki, Finland \\ ${ }^{c}$ Physics Department, University of Crete, \\ P.O. Box 2208, 71003 Heraklion, Crete, Greece ${ }^{2}$ \\ ${ }^{d}$ APC, Université Paris 7 , \\ Bâtiment Condorcet, 75205, Paris Cedex 13, France $^{3}$ \\ e Theory Group, Physics Department, CERN, \\ CH-1211, Geneva 23, Switzerland ${ }^{4}$ \\ ${ }^{f}$ Department of Physics, University of Helsinki, \\ P.O. Box 64, FI-00014 Helsinki, Finland \\ E-mail: timo.s.alho@jyu.fi, mjarvine@physics.uoc.gr, \\ keijo.kajantie@helsinki.fi, rosen@physics.uoc.gr, \\ kimmo.i.tuominen@helsinki.fi
}

ERRATUM TO: JHEP04(2014)124

ARXIV EPRINT: 1312.5199

\footnotetext{
${ }^{1}$ http://hep.physics.uoc.gr/ kiritsis/.

${ }^{2}$ http://hep.physics.uoc.gr/.

${ }^{3}$ http://www.apc.univ-paris7.fr/APC_CS/.

${ }^{4}$ http://wwwth.cern.ch/.
} 


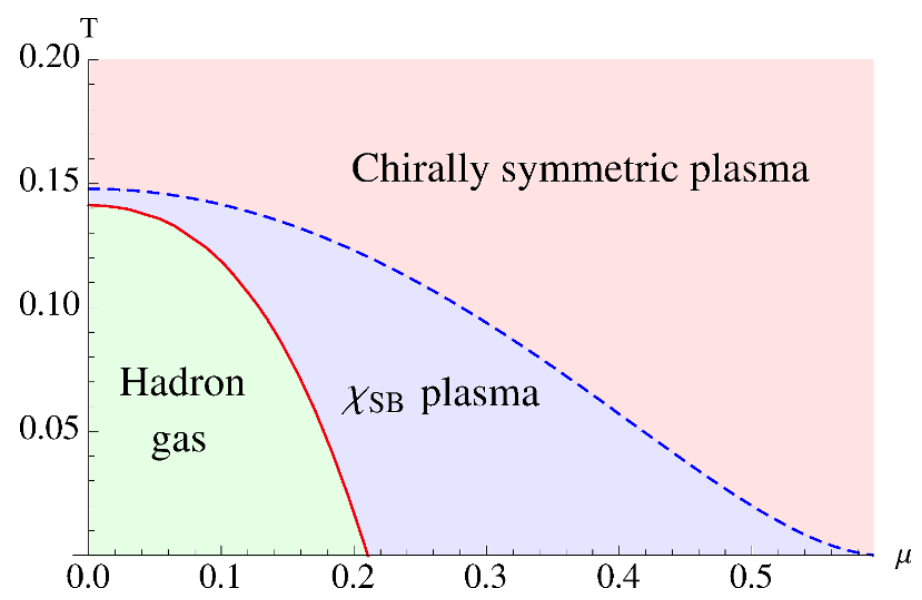

Figure 1. Chemical potential dependence of transition temperatures of the deconfining $\left(T_{h}(\mu)\right)$ and chiral $\left(T_{\chi}(\mu)\right)$ transitions at $m_{q}=0$. The dashed line corresponds to a second order phase transition while the solid line corresponds to a first order transition. The main correction outlined in this errata is that the critical point and first order transition at large $\mu$ disappear, and the chiral symmetry restoring transition remains second order all the way to the lowest temperatures computed.

After the publication of the paper [1], it was discovered that the numerical code used for computing the phase diagram had an error. Specificially, the $(1-K)$ term in $(2.32)$ was accidentally replaced by 1 in the code. Since $K$ is proportional to $\tilde{n}^{2} \tau^{\prime 2}$, omitting it in principle causes an error in all calculations involving the chiral symmetry breaking phase at any finite $\tilde{n}$, or equivalently, finite $\mu$. However, at small values of $\tilde{n}$ the error is small and becomes significant only around $\tilde{n} \sim 7, \mu \sim 0.3$. Furthermore, at large $\tilde{n}, \tilde{n}>20$, the error is again small.

We also note that the error only affects the computation of the solutions corresponding to the chiral symmetry breaking phase. All calculations referring to the chirally symmetric phase are unaffected, including in particular the discussion concerning the quantum critical $A d S_{2}$ regime at $T=0$. The general structure of the results and the calculational methods in this model are also unaffected.

The primary effect of correcting this error is that the critical point in figure 1 disappears, and instead the chiral symmetry restoring transition continues as second order all the way to the smallest values of $T$ studied. This invalidates the discussions which refer to the tricritical point and the first order part of the chiral symmetry restoring transition, primarily on pages 3, 29-31 and 37, together with appendices D and E. In addition to figure 1, figures $7,11,13,14$ and 15 are affected. The corrected main plots, corresponding to figure 1 and figure 7 in the original paper, are exhibited in figure 1 and figure 2, respectively. ${ }^{1}$

\footnotetext{
${ }^{1}$ The corrected full paper is available as arXiv V2 of [1].
} 


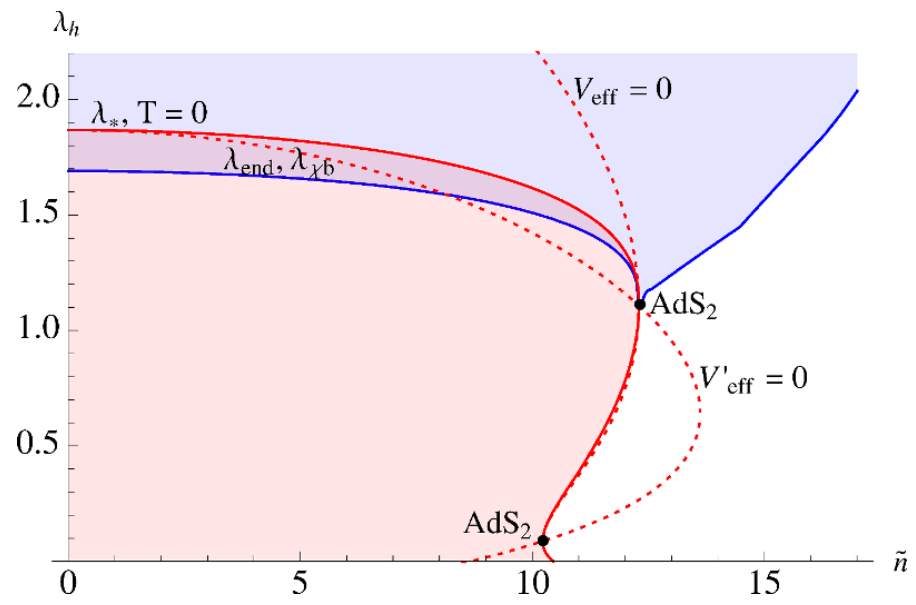

Figure 2. The physical region on the $\lambda_{h}, \tilde{n}$ plane for chirally symmetric (red region) and chirally broken (blue region, unbounded above) solutions. The chirally symmetric region is bounded from above by the curve $\lambda_{*}(\tilde{n})$ along which $T=0$ up to the point $\operatorname{AdS}_{2}$ at $\tilde{n}=12.295, \lambda_{h}=1.108$, then from the right by a segment of the curve $V_{\text {eff }}=0$ up to the second $\mathrm{AdS}_{2}$ point at $\tilde{n}=10.223, \lambda_{h}=0.0873$ and finally by a segment to $\tilde{n}=10.457, \lambda_{h}=0$. Tachyonic chiral symmetry breaking solutions exist only above the blue curve $\lambda_{\text {end }}(\tilde{n})$. In this corrected version of the plot, the curve has no discontinuity, and it goes through the $\mathrm{AdS}_{2}$ point. The chiral symmetry restoring transition lies entirely on the segment of the $\lambda_{\text {end }}(\tilde{n})$ curve between $\tilde{n}=0$ and the $\mathrm{AdS}_{2}$ point. The dashed lines are $V_{\text {eff }}=0$ and $V_{\text {eff }}^{\prime}\left(\lambda_{h}\right)=0$ at $\tau=0$.

Open Access. This article is distributed under the terms of the Creative Commons Attribution License (CC-BY 4.0), which permits any use, distribution and reproduction in any medium, provided the original author(s) and source are credited.

\section{References}

[1] T. Alho et al., A holographic model for QCD in the Veneziano limit at finite temperature and density, JHEP 04 (2014) 124 [arXiv:1312.5199] [INSPIRE]. 